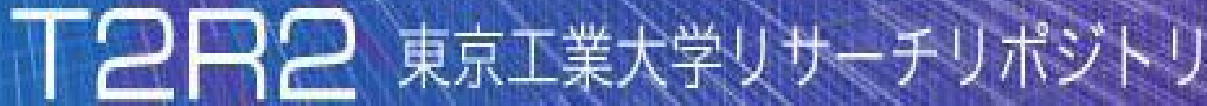

\section{Tokyo Tech Research Repository}

\section{論文 /著書情報 \\ Article /Book Information}

\begin{tabular}{|c|c|}
\hline Title & Armrest Joystick - Mechanism Design and Basic Experiments \\
\hline Author & Hiroaki Ishida, Tetsuo Hagiwara, Koji Ueda, SHIGEO HIROSE \\
\hline Journal/Book name & , Vol. 25, , pp. 5119-5124 \\
\hline Issue date & 2012,10 \\
\hline DOI & http://dx.doi.org/10.1109/RO S.2012.6385981 \\
\hline URL & http://www.ieee.org/ndex.html \\
\hline Copyright & $\begin{array}{l}\text { (c) } 2012 \text { IEEE. Personal use of this material is permitted. Permission } \\
\text { from IEEE must be obtained for all other users, including } \\
\text { reprinting /republishing this material for advertising or promotional } \\
\text { purposes, creating new collective works for resale or redistribution to } \\
\text { servers or lists, or reuse of any copyrighted components of this work in } \\
\text { other works. }\end{array}$ \\
\hline Note & $\begin{array}{l}\text { このファイルは著者 (最終) 版です。 } \\
\text { This file is author (final) version. }\end{array}$ \\
\hline
\end{tabular}




\title{
Armrest Joystick -Mechanism Design and Basic Experiments-
}

\author{
Hiroaki Ishida, Tetsuo Hagiwara, Koji Ueda and Shigeo Hirose
}

\begin{abstract}
Manipulators, which are mounted on robots that substitute man at dangerous places like indoors and outdoors disaster sites or mine fields, have been mainly controlled by joysticks. However, joysticks are difficult to manipulate and complex tasks become hard to be done by operators because of a limit and discordance of controlled degree of freedom. Therefore, a controller with high operability like the MasterSlave system is needed, nevertheless conventional controllers are not used in these situations because there is no controller which has compact structures and intuitively controls $3 \mathrm{DOF}$ position and 3DOF posture. In this research, we propose a remote controller "Armrest Joystick" of the Master-Slave system which has high portability and operability. The Armrest Joystick is attached to a foldable portable chair and can be carried easily. It consists of a 3DOF position controller with an armrest supporting the operator's arm during operations, a 3DOF posture controller and a gripper controller. Furthermore, it has motors for the force feedback on all axes for more intuitive operations. In this paper, we report the basic concept and design of the Armrest Joystick, and the experiments which verify operability and the validity of the force feedback.
\end{abstract}

\section{INTRODUCTION}

In disaster sites, such as outdoors and buildings damaged by terror attacks or natural disasters, robots that can gather information and manage various works before humans are necessary. Moreover, in mine fields robots which investigate and remove mines are urgently needed.

In order to automate all these tasks, robots need to be adapted to the severe and various environments, and the use of advanced control and sensing is essential. In this context, advanced operations with robotic arms are currently done by human's remote operation. In recent years, "PackBot" of iRobot [1] managed various tasks by remote operation with the joystick in Fukushima Daiichi Nuclear Power Station after the Tohoku Earthquake, 2011[2]. Although the joystick is now widely used for these remote operations, it is assumed that the use of robotic arms will be expanded to more complex and different applications, so the introduction of a controller of the Master-Slave system which can be operated with higher operability is indispensable[3]. The Master-Slave system operates the arm by the side of the operator, and its operability is very high. However, the Maste-Slave system controllers can not be used in a wide range of hazardous areas and the joystick controllers can be used widely because the former controllers are large-sized and have no portability for operability[4][5]. Moreover, although it is also known that higher operability can be acquired by a force feedback, it makes the portability even more problematic. Furthermore,

Department of Mechanical and Aerospace Engineering Tokyo Institute of Technology 2-12-1 O-okayama Meguro-ku, Tokyo 152-8552, Japan ishida@robotics.mes.titech.ac.jp

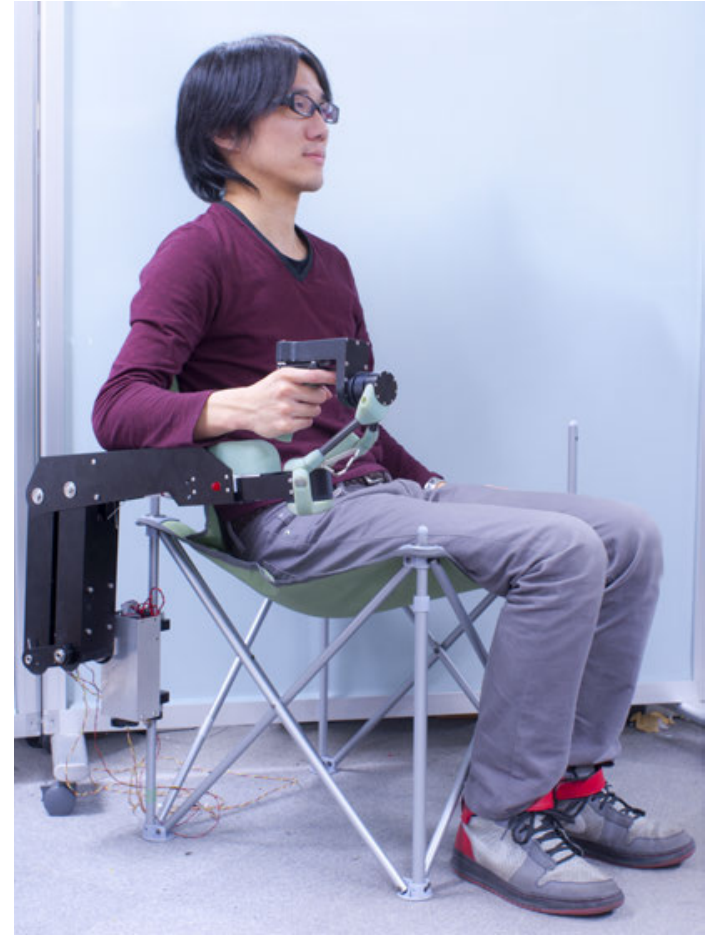

Fig. 1. Armrest Joystick

since in most of them the operator's arm has to be kept floating in the air, it is also hard to do prolonged operations without fatigue and to move their hand stably in delicate operations. Finally, once the operability of the arm and the wrist controllers are prioritized, the gripper controller's design hasn't yet been analyzed as deeply as other parts.

In this research, we propose the bilateral remote controller, "Armrest Joystick," of the Master-Slave system with the armrest characterized by high portability and operability(Fig.1) and develop the operation mechanisms for the robotic arm and the gripper.

\section{SLAVE ROBOT}

In the Armrest Joystick, we consider as the slave robots, the rescue robot "Helios IX"(Fig.2(a)) [6] and the humanitarian demining robot "Gryphon"(Fig.2(b)) [7] developed in our laboratory. Both robots have a $6 \mathrm{DOF}$ arm, and a $1 \mathrm{DOF}$ gripper at their tip. Helios IX's arm is currently controlled by a "Wii Controller" [8] of a 2DOF joystick and buttons, and Gryphon's arm by two 3DOF joysticks and buttons. By these controllers mainly consisting of a joystick, Helios IX can open and close doors, and Gryphon can remove vegetation by remote control. However, when doing more complicated and delicate works like using tools and digging mines, a controller for the Master-Slave system capable of controlling intuitively and several axes simultaneously is necessary. 


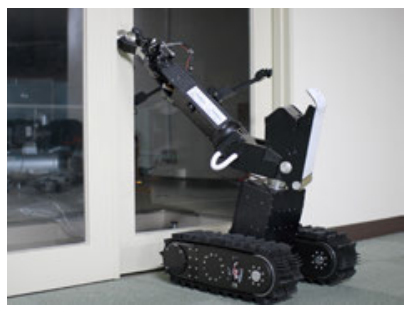

(a) Helios IX
Fig. 2. Slave Robots

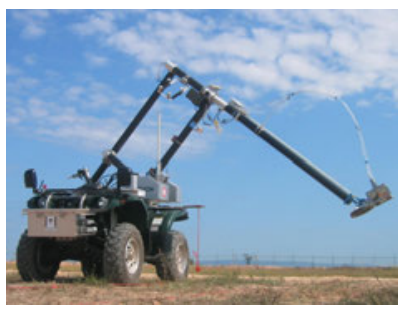

(b) Gryphon

\section{BASIC DESIGN CONCEPT}

First, we present basic design concepts of the controller.

1) The controller's size and weight is within a range in which the operator can carry it by himself.

2) It has the widest movable range as possible.

3) It has a mechanism that can be operated lightly without receiving resistance by the controller's weight.

4) It returns force and torque from the slave robot, and performs a bilateral control.

5) It has a low reduction-ratio gear box that can be lightly operated without receiving friction resistance from the gear box as possible.

Regarding the number of degrees of freedom for the controller, the arm's controller requires more than 6 and the gripper's controller requires more than 1 in order to operate all degrees of freedom on the slave's robot. Increasing the degrees of freedom permits a controller which can follow all kinds of motion of the operator's arm or wrist. However, it will directly increase the size and the weight. Therefore, the arm's controller constitutes of the necessary 6DOF considering portability. Regarding their arrangement, it is adopted the different structural form since it is easy to decrease the whole mechanism and perform scale conversions of force and torque[3].

Based on these basic concepts, we performed detailed individual considerations for the 3DOF position controller operated by the operator's arm, the 3DOF posture controller by the operator's wrist, and the gripper controller by the operator's fingers.

\section{3DOF POSITION CONTROLLER}

\section{A. Concept of 3DOF Position Controller}

The position controller occupies the most part of the mechanism, and has to be moved by the operator's arm during operations. If the position controller requires a heavy load to the operator's arm, the operator would get exhausted soon even after short operations, and operation mistakes would be increased. In daily tasks, a man usually rests the arm on the body, a desk or an armrest of a chair when doing various works, and for this reason we propose to introduce an armrest into the controller for supporting the operator's whole arm. Furthermore, we also consider that it keeps the operator's arm stable during delicate operations. In controllers for field robots, an armrest is not introduced in order to maximize the operation range, however some examples exist in controllers for surgical operations, da Vinci and so on[9][10]. A narrow moving range is enough for these controllers because the range of the robot itself is also narrow. Regarding the movable range, the controller and the armrest are usually fixed on a desk in front of the operator, and the operation range depends on the size of the desk. Furthermore, since the controller is in fact fixed on the ground when receiving counter force and torque from the force feedback, there is a problem that a certain amount of the size and the weight are required for the desk. Therefore, another fixing method is needed for these problems.

In this paper, we propose a controller whose linkages include the armrest, which supports the operator's arm in Fig.3 and are fixed to the foldable chair for outdoor operations. Since the foldable chair for outdoor is compact and lightweight, and can be carried easily, it is the practical base for the controller used in the field. Even if the foldable chair is small and lightweight, it is sufficient because the operator's weight can also com-

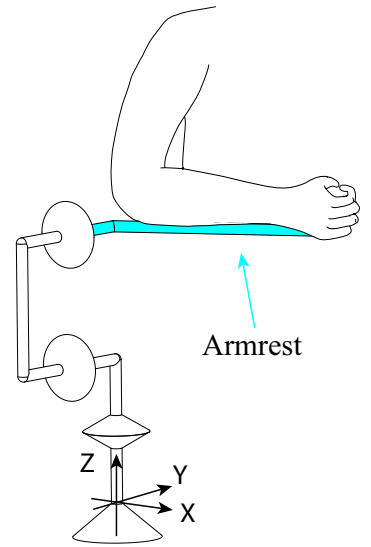

Fig. 3. Concept of the Position Controller pensate the counter force and torque. During operations the operator doesn't have to keep standing, and it is possible for the operator to control it for a long time. By moving the armrest itself as the controller's linkage, we consider that it can get a wider movable range around them. In the previous work, Yokokohji et al. studied the controller of "T-52 Enryu Re" with an armrest[11]. However, portability is not taken into consideration. Furthermore, the armrest mechanism supports only the elbow, and is considered as switches of the redundant degrees of freedom of the slave's manipulator. In our proposed controller, we consider that the operator can carry it easily and operate for a longer time without fatigue because the armrest supports the operator's whole arm.

Furthermore, having just the controller with armrest, the only operator's arm must support the weight of the controller. Therefore, the operator would be tired without the ability to move it lightly. Besides, motors need more power for not only the force feedback but also moving the controller's weight itself, and it causes the increase of the weight and the size of motors. Therefore, we adapt the weight compensation mechanism to the controller. For the weight compensation, a counter-weight or a spring is commonly used. In the counter-weight method, the weight of the mechanism can be held regardless of its posture. However, there are some problems that the counter-weight itself leads to the increase of the weight of the controller and the operation range becomes narrow due to interferences between the controller and the counter-weight. Moreover, sudden acceleration and deceleration become difficult and the load on the motors increases due to the inertia increasing of the mechanism. On the other hand, in the spring method these problems can 
be eliminated, however the range of the weight compensation is limited depending on its posture. Recent researches show a method with a spring and a non-circular pulley that can compensate the weight of the mechanism regardless of its posture.

In this controller, the non-circular pulley and the spring constitute the mechanism for compensating the controller's weight in the wide movable range.

\section{B. Design of 3DOF Position Controller}

Fig.4(a) shows the 3DOF position controller. The foldable chair used as a base has a light weight of approximately $3.8 \mathrm{~kg}$ and it can be compactly folded up for transport. The controller is fixed to the lower part of the lear leg of the chair, and the counter force of the force feedback can be definitely compensated with the operator's weight.

It consists of $1 \mathrm{DOF}$ with the perpendicular axis in the leg and 2DOF parallel linkages. The tip of the parallel linkages has the function of an armrest. These mechanisms permits 3DOF position control(Fig.5). By using the parallel
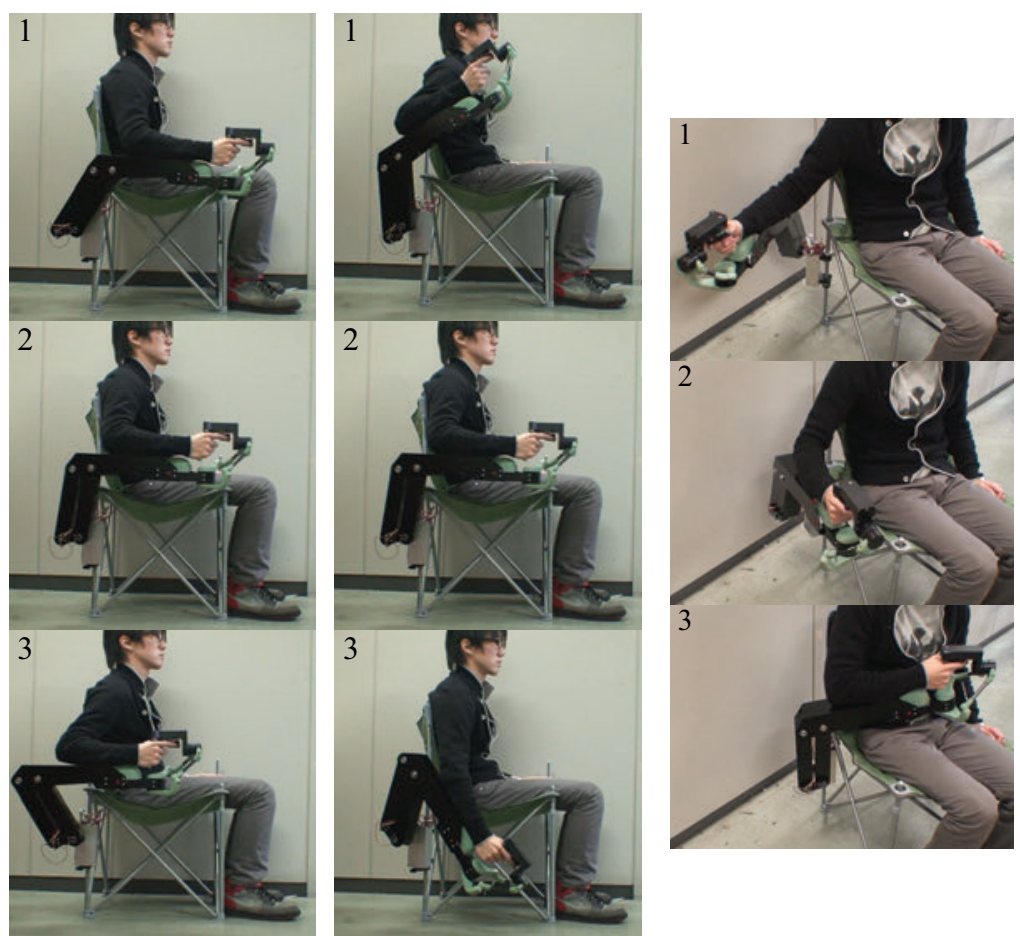

(a) Front and Back Motion (b) Upward and Downward (c) Right and Left(Yaw) Motion Motion

Fig. 5. Motion of the Position Controller

\section{3DOF POSTURE CONTROLLER}

as Fig 4(b) around the root of the parallel linkages, and it succeeds in the weight reduction of the link at the tip and light operations. Inside the link, all springs and pulleys for the weight compensation were arranged for not exposing outside, and the non-circular pulley is especially used for the compensation of the link at the tip. It works in a wide range of the weight compensation regardless of its posture. The details of them are explained in the presentation.

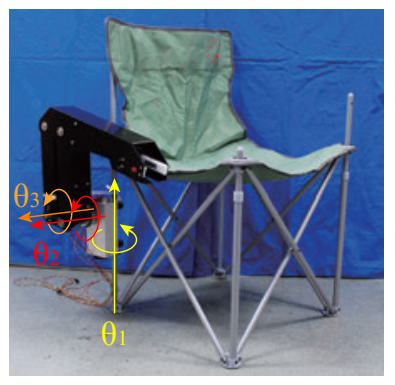

(a) Overview

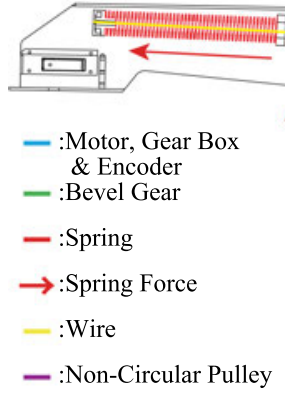

(b) Cross Section

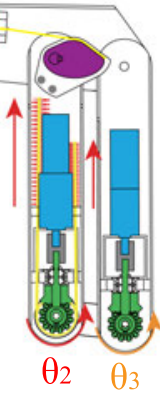

$\theta_{2} \quad \theta_{3}$

TABLE I

SPECIFICATION OF 3DOF POSITION CONTROLLER

\begin{tabular}{|c|c|}
\hline Weight & $7.6 \mathrm{~kg}$ (including Chair) \\
\hline Size & $359 \times 186 \times 407 \mathrm{~mm}$ (without Chair) \\
\hline Move Range & Radius: $200 \sim 400 \mathrm{~mm}$ Height: $450 \mathrm{~mm}$ Cylinder \\
& Yaw: $-60 \sim 60^{\circ}$ \\
\hline Motor Power & $11 \mathrm{~W}$ (Each Axis) \\
\hline Reduction Ratio & $\theta_{1}: 111: 1, \theta_{2}: 246: 1, \theta_{3}: 128: 1$ \\
\hline Feedback Force & $3 \mathrm{~N}$ (Each Axis) \\
\hline
\end{tabular}

\section{A. Concept of 3DOF Posture Controller}

In the posture controller, the previous work demonstrates that the rotation center of the controller mechanism should coincide the center of the operator's hand for an effective operation[13]. One of this mechanism is the gimbal mechanism. When we make the gimbal mechanism by simple linkages, we usually use the serial linkage mechanism[14]. However, there is a problem that the weight of the mechanism becomes a resistance to the hand because some motors for the force feedback are attached to the linkages. In order to solve this problem, a wrist mechanism with the parallel linkage is effective[13]. However, the joint arrangement and the length of the links may decrease the movable range.

Thus, we propose a mechanism in which the moving range of the controller is wider than that of the operator's wrist by contriving the arrangement of the joints of the controller.

\section{B. Design of 3DOF Posture Controller}

Fig.6(a) shows the 3DOF wrist mechanism. It consists of a 2DOF spherical parallel link mechanisms and a 1DOF rotation mechanism at the tip of the parallel linkage. The axes of rotation of joints of spherical linkages and the 1DOF mechanism at the tip intersect in one point around the center of the operator's grip, and follow the gimbal mechanism concept. By using the parallel linkage, the motors are removed on the movable linkages and are arranged on the base of the posture controller(Fig.6(b)).

Regarding the movable range of the parallel link, arranging the rotation axes of the root at the same axis, unlike usual 
arrangement, provides the parallel link with a wide movable range. Since the $1 \mathrm{DOF}$ mechanism at the tip is independent from the parallel linkage mechanism, it has a wide movable range. These two axes mechanism are arranged each on Yaw and Roll axis which require a wide movable range for the wrist and can fill wider than the movable range of the operator's wrist(Fig.7).

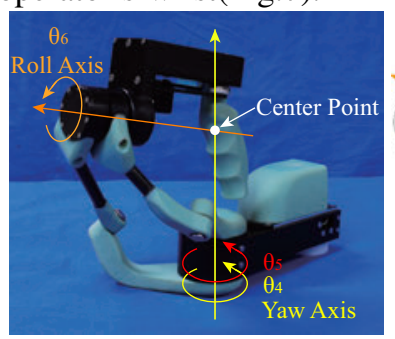

(a) Overview

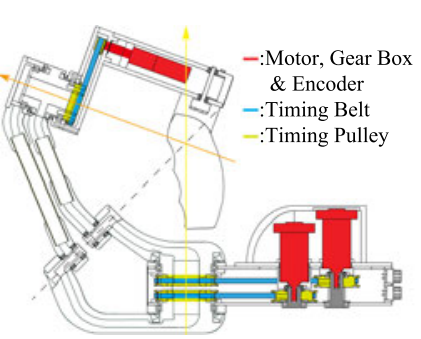

(b) Cross Section Fig. 6. 3DOF Posture Controller

TABLE II

SPECIFICATION OF 3DOF POSTURE CONTROLLER

\begin{tabular}{|c|c|}
\hline Weight & $1543 \mathrm{~g}$ \\
\hline Size & $310 \times 155 \times 211 \mathrm{~mm}$ \\
\hline Move Range & Roll: $-112 \sim 111^{\circ}$ \\
& Pitch: $-55 \sim 20^{\circ}$ \\
& Yaw: $-155 \sim 150^{\circ}$ \\
\hline Motor Power & Roll: $12 \mathrm{~W}$ \\
& Pitch \& Yaw: $6 \mathrm{~W}$ \\
\hline Reduction Ratio & $\theta_{4} \& \theta_{5}: 19: 1, \theta_{6}: 17: 1$ \\
\hline Feedback Torque & $0.02 \mathrm{Nm}$ (Each Axis) \\
\hline
\end{tabular}

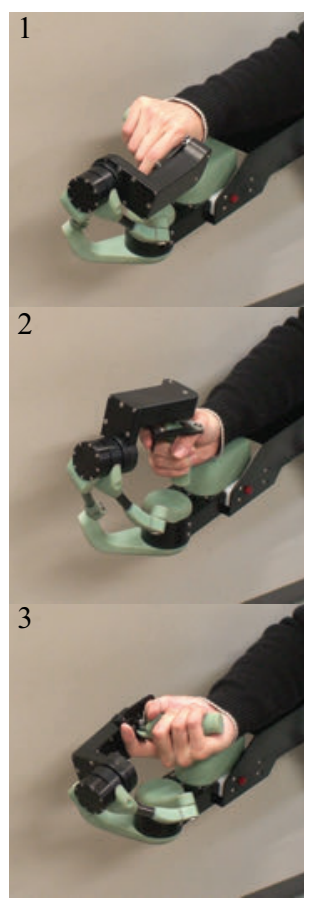

(a) Roll Motion

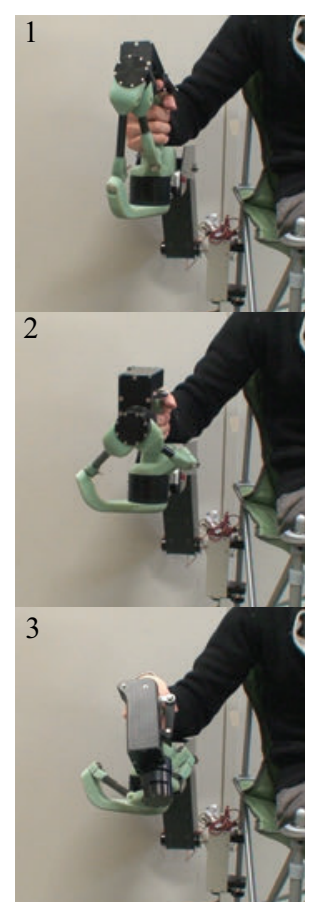

(b) Pitch Motion

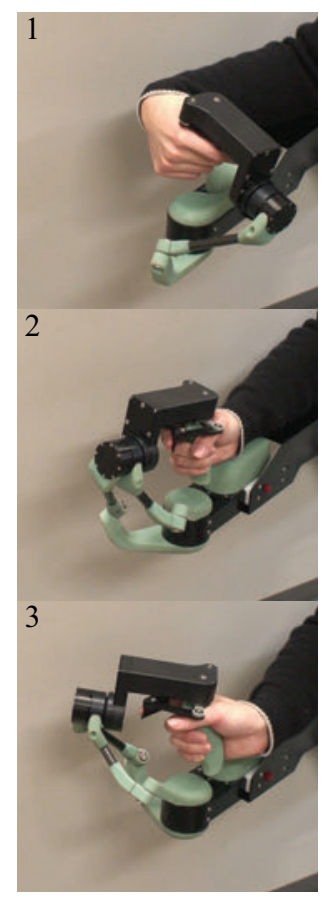

(c) Yaw Motion
Fig. 7. Motion of the Posture Controller

\section{GRIPPER CONTROLLER}

\section{A. Concept of Gripper Controller}

Since the gripper controller is a portion which contacts objects through the gripper of the slave robot, it needs to follow the complicated motion of the fingers in order to perform certain operations. Therefore, the linkage mecha- nism is desirable as much as other operation mechanisms because operability of buttons or triggers (which are used mostly) is not satisfactory in delicate works, especially with the force feedback. For example, in the linkage mechanism with simple 1DOF, the link and a finger become slippery without having the same rotation axis, and operability is compromised(Fig.8). Therefore, a mechanism that keeps the same axes of rotation for the link and the finger, and need to follow enough movements of the finger.

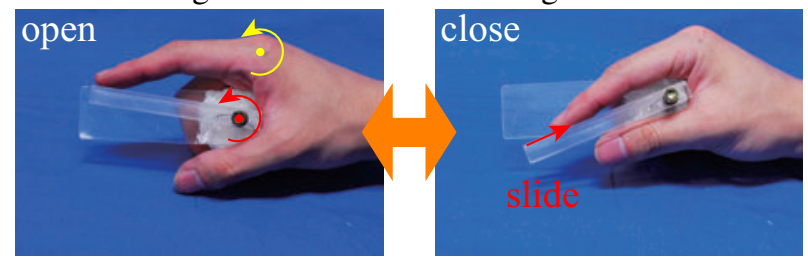

Fig. 8. Problem of the Simple Finger Link

Even if such mechanism is done, operability is affected by arrangement of the mechanism. In operations which the mechanism is used with uncomfortable postures (such as moving the wrist to outside or opening too much the finger), it can be imagined that the wrist and the finger get tired easily, and it doesn't have enough operability. For this reason, it is also necessary to consider the arrangement without overloading the wrist and the finger.

We propose a gripper controller in order to achieve smooth operations which fit to the position of the finger and the wrist can follow the movement of the finger by using the exoskeleton linkage mechanism, which keeps the same rotation axes for the finger and mechanism.

\section{B. Design of Gripper Controller}

The gripper operation mechanism consists of twobar exoskeleton linkages along the index finger and two-bar linkages connected to the tip of them(Fig.9(a)). The joints of the exoskeleton linkages are arranged on the MP joint and the PIP joint of the index finger in order to follow the movement of fingers when holding between the index finger and the thumb. Other twobar linkages connected to the exoskeleton linkage have the function measuring the position of the fingerlink and returning force. All mechanisms were arranged considering the usual posture of the operator's wrist and fingers. Two base joints of all linkages have springs, and they output torque by pressing the whole link against the index finger. Although it has no wearing mechanism, the linkages can smoothly follow the movement of the index finger(Fig.10). This mechanism has only one motor for the force feedback and one encoder for the angle measurement of the termination of the motor in the upper part of the gripper operation mechanism although it is a 2DOF mechanism (Fig.9(b)). On the other hand, the movement of the finger joints has correlations when openingand-closing, and so the trajectory at the tip of the index finger can be estimated with one encoder. It performs the force feedback by the torque of the spring and the motor.

Furthermore, when using the force feedback, the motion 
which releases the finger to the outside can be made by the remaining $1 \mathrm{DOF}$, and it also prevents operating to grasp an object too tightly.

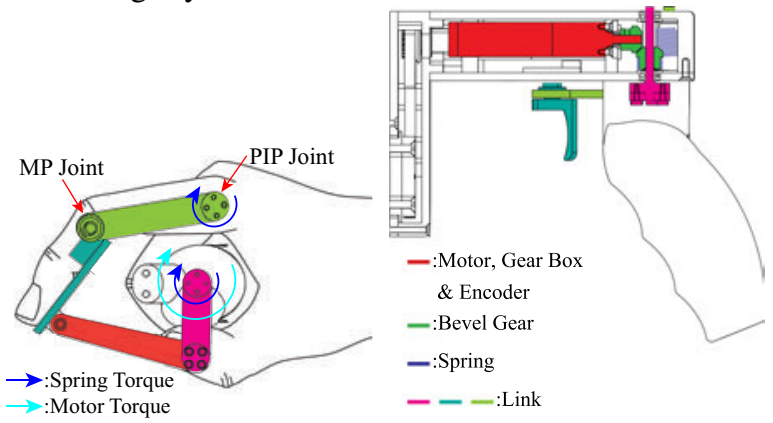

(a) Overview

(b) Cross Section

Fig. 9. Gripper Controller

TABLE III

SPECIFICATION OF GRIPPER CONTROLLER

\begin{tabular}{|c|c|}
\hline Motor Power & $2 \mathrm{~W}$ \\
\hline Reduction Ratio & $17: 1$ \\
\hline Feedback Force & $1 \mathrm{~N}$ \\
\hline
\end{tabular}

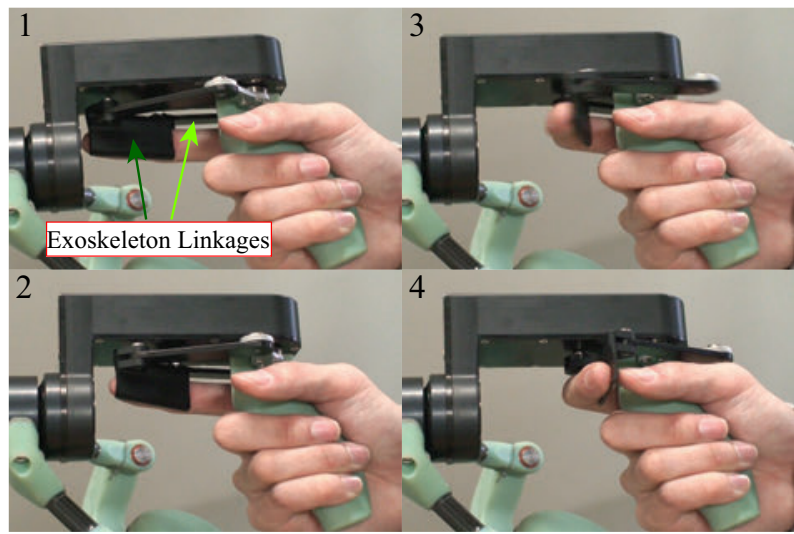

Fig. 10. Motion of the Gripper Controller

\section{ARMREST JOYSTICK}

The controller "Armrest Joystick" consists of a 3DOF position controller with an armrest attached to the foldable chair, and a 3DOF posture controller and a gripper controller which are attached at the tip of the position controller(Fig.1). The weight of all mechanism is approximately $9.1 \mathrm{~kg}$. After removing the posture controller and folding the chair,

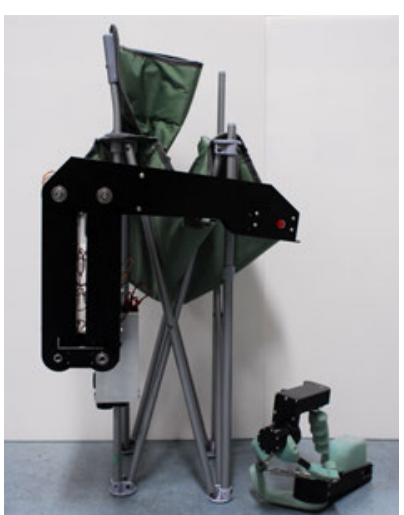

Fig. 11. Mobile Form the size and the weight is within a range in which the operator can transport it by himself(Fig.11). The posture controller is easily attached and removed mechanically and electrically from the position controller. All of the motor controllers and power supplies are not placed on the Armrest Joystick, but at present we have developed them mounted on the Armrest Joystick.

\section{EXPERIMENTS}

\section{A. Operability Experiment}

We conducted a first experiment to remotely control Helios IX with the Armrest Joystick. The reason why we selected Helios IX is that Helios IX has been studied for long years and the reliability as a slave robot is very high. In this experiment, we used a simple unilateral control system which, first, each motor controller reads the value of an encoder on each motor, and then a PC calculates the 6DOF position and posture of the grip and the angle of the gripper controller of the Armrest Joystick, finally operating the position and posture of the tip of the Helios IX's arm and the gripper.

We confirmed that 3DOF position, 3DOF posture, and the gripper could be intuitively operated and we could finish a easy task which Helios IX placed an plastic bottle from one height to another(Fig.12).

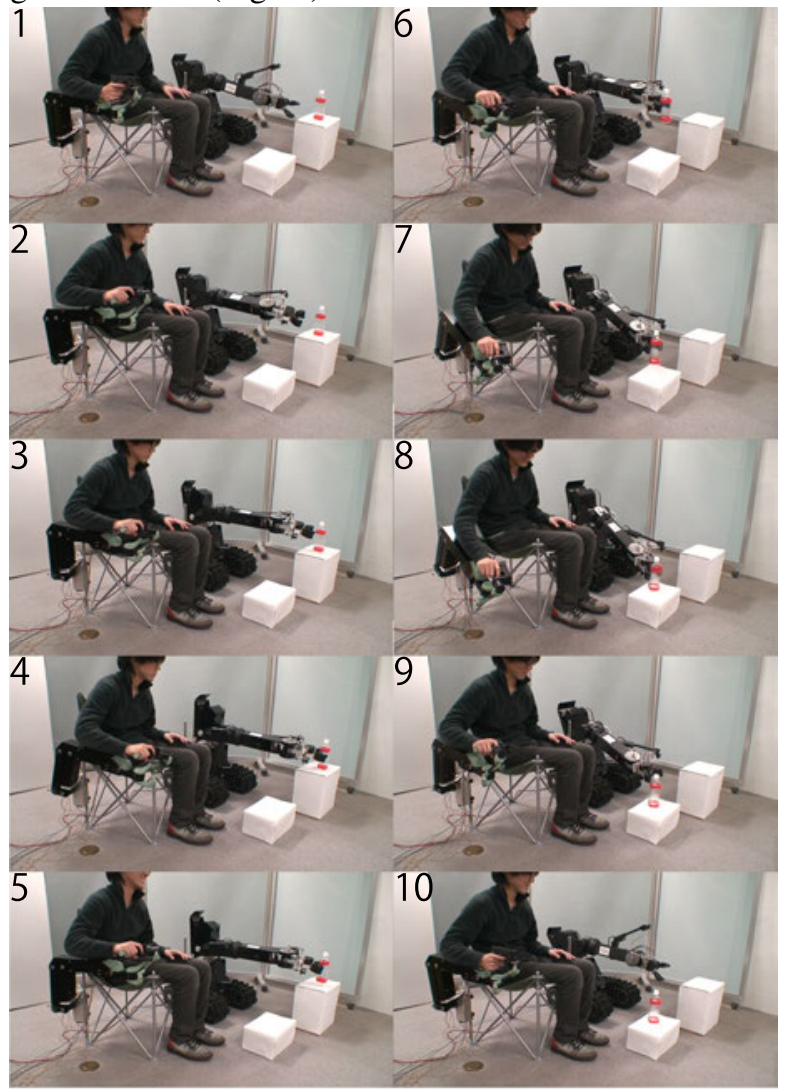

Fig. 12. Basic Move Experiment

We conducted a simple experiment for the effect of the weight compensation and the armrest. The experiment consisted of operators who controlled Helios IX with and without the weight compensation and the armrest.

We confirmed that operators could move without getting tired and operate stably thanks to the weight compensation and the armrest compared to the case without them. Moreover, we could operate it for only $2 \sim 3$ minutes without the weight compensation. The result is achieved by the only operators' feeling, so we have to verify the effect of the weight compensation and the armrest in details in the future. 


\section{B. Comparison with the Wii Controller}

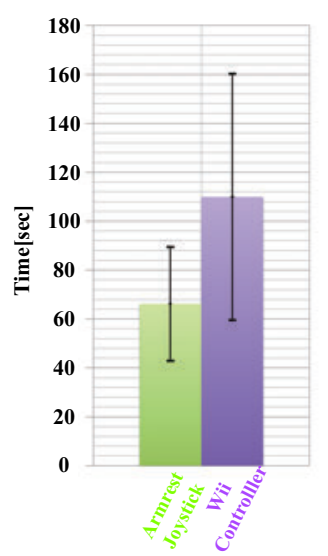

We conducted the experiment to compare operability with the Wii Controller of the conventional joystick and the Armrest Joystick. Five subjects, who operate Helios IX first, used both controllers to place an aluminium can with weights inside from one height to another. This operation was repeated more than 4 times and the time was measured.

Fig.13 shows the averaged time of each controller. We confirmed that the Armrest Joystick reduced the time for this task compared with Wii controller. It suggests

Fig. 13. Average Time

that they can operate the robotic arm with high operability by using the Armrest Joystick. However, we have to compare other types of controllers with the Armrest Joystick regarding operability.

\section{Force Feedback Experiment}

We conducted the experiment for confirming the validity of the force feedback. So far, the force feedback has been implemented only on the gripper controller, and the force reflection type bilateral control system has been adopted. In the experiment, we use the current of the gripper's motor of the Helios IX as a force sensor in a slave robot. The subjects were the same as the former experiment, and the trial was done 2 times each. We compared the current of the gripper's motor of Helios IX with and without the force feedback when Helios IX moved the can up. Fig. 14 shows the current and the count of the hall effect sensor of the gripper's motor of Helios IX by the existence of the force feedback, and Fig.15 shows the averaged current. In the no force feedback case, operators had to visually judge the gripping force, and they ended up inputting command for too much force for not letting the can fall. With the force feedback, their fingertip could judge the feedback force and input proper commands required to raise without crushing the aluminum can.

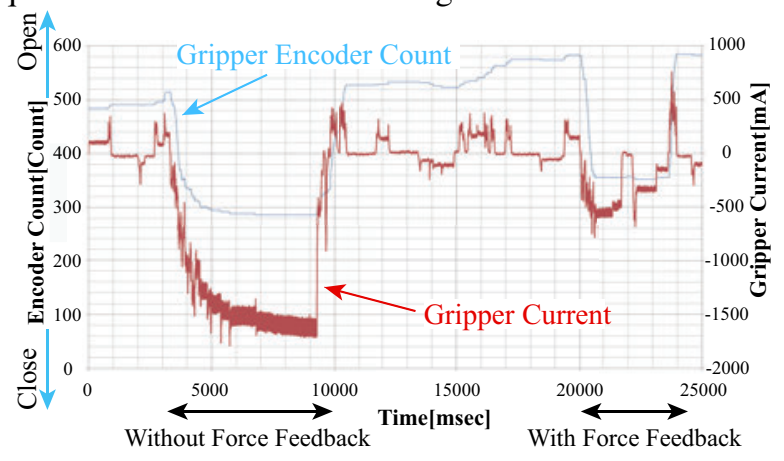

Fig. 14. Current Data \& Encoder Count

\section{CONCLUSIONS}

In this paper, we proposed the concept of the controller "Armrest Joystick" with high portability and operability

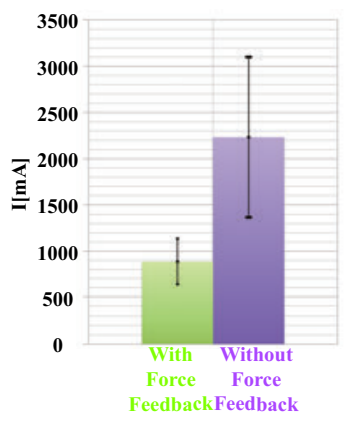

Fig. 15. Average Current

for the robots used in fields, such as disaster sites and mine fields. We studied the design of each mechanism and developed the Armrest Joystick. Furthermore, by the basic experiments, we succeeded in achieving higher operability than the Wii Controller, and confirmed the validity of the force feedback in the gripper controller.

We plan to implement the force feedback on the position controller and the posture controller, and to apply the Armrest Joystick to Gryphon's system. We also plan to do experiments to analyze the validity of operability.

\section{REFERENCES}

[1] iRobot Corporation, http://www.irobot.com.

[2] Tokyo Electric Power Company, http://www.tepco.co.jp/en/indexe.html.

[3] Robotics Society of Japan, Robotics Handbook, 2005. (in Japanese)

[4] H. Hasunuma, H. Kagaya, M. Takatori, J. Fujimori, F. Mifune, S. Shikoda, M. Kobayashi, T. Itoko, S. Tachi, Development of Teleoperation Master System with a Kinesthetic Sensation of Presence, Proceedings of the 9th International Conference on Artificial Reality and Tele-existence '99. pp.53-59, Tokyo, 1999.

[5] J. M. Hollerbach, S. C. Jacobsen, Anthropomorphic robots and human interactions, Proceedings of the 1st International Symposium on Humanoid Robots, pp.83-91, 1996.

[6] K. Ueda, M. Guarnieri, R. Hodoshima, E. F. Fukushima, S, Hirose, Improvement of the Remote Operability for the Arm-Equipped Tracked Vehicle HELIOS IX, Proceedings of the 2010 IEEE/RSJ International Conference on Intelligent Robots and Systems, pp.363-369, 2010.

[7] T. Matsuzawa, E. F. Fukushima, S, Hirose, Research on humanitarian demining robot "Gryphon" -Development of gripper and cutter mechanism for vegetation clearance-, Proceedings of the 2007 SICE Conference on System Integration, pp.1031-1032, 2007. (in Japanese)

[8] Nintendo, http://www.nintendo.com/?country=USlang=en.

[9] Intuitive Surgical, http://www.intuitivesurgical.com.

[10] G. Tholey, J. P. Desai, Design and Development of a General Purpose 7 DOF Haptic Device, Symposium on Haptic Interfaces for Virtual Environment and Teleoperator Systems 2006, pp.95-101, 2006.

[11] S. Ito, Y. Yokokohji, Maneuverability of Master Control Devices Considering the Musculo-skeletal Model of an Operator, Euro Haptics conference and Symposium on Haptic Interfaces for Virtual Environment and Teleoperator Systems, pp.57-62, 2009

[12] G. Endo, H. Yamada, A. Yajima, M. Ogata, S. Hirose. A passive weight compensation mechanism with a non-circular pulley and a spring, Proceedings of the 2010 IEEE International Conference on Robotics and Automation, pp.3843-3848, 2010.

[13] K. Nakashima, R. Ienaka, J. Fujimori, H. Kagaya, H. Moriyama, H. Hasunuma, T. Kubota, S. Shikoda, The Development of the Portable Master Control System (HR Operating Industrial Vehicles), Proceedings of the JSME Conference on Robotics and Mechatronics, Vol.20, pp.2D16, 2002. (in Japanese)

[14] S. Nakagawara, R. Tadakuma, H. Kajimoto, N. Kawakami, S. Tachi, A Method to Solve Inverse Kinematics of Redundant Slave Arm in The Master-Slave System with Different Degrees of Freedom, Proceedings of the 2004 International Symposium on Measurement, Control, and Robots, A12, 2004 